\title{
Cloaking a sensor via transformation optics
}

\author{
Allan Greenleaf, ${ }^{1}$ Yaroslav Kurylev, ${ }^{2}$ Matti Lassas, ${ }^{3}$ and Gunther Uhlmann ${ }^{4,5,}{ }^{*}$ \\ ${ }^{1}$ Department of Mathematics, University of Rochester, Rochester, New York 14627, USA \\ ${ }^{2}$ Department of Mathematical Sciences, University College London, London, WC1E 6BT, United Kingdom \\ ${ }^{3}$ Department of Mathematics, University of Helsinki, FIN-00014, Finland \\ ${ }^{4}$ Department of Mathematics, University of California, Irvine, Irvine, CA 92697, USA \\ ${ }^{5}$ Department of Mathematics, University of Washington, Seattle, WA 98195, USA
}

(Received 3 December 2009; revised manuscript received 31 October 2010; published 21 January 2011)

\begin{abstract}
Ideal transformation optics cloaking at positive frequency, besides rendering the cloaked region invisible to detection by scattering of incident waves, also shields the region from those same waves. In contrast, we demonstrate that approximate cloaking permits a strong coupling between the cloaked and uncloaked regions; careful choice of parameters allows this coupling to be amplified, leading to effective cloaks with degraded shielding. The sensor modes we describe are close to but distinct from interior resonances, which destroy cloaking. As one application, we describe how to use transformation optics to hide sensors in the cloaked region and yet enable the sensors to efficiently measure incident waves on the exterior of the cloak, an effect similar to the plasmon-based approach of Alù and Engheta.
\end{abstract}

DOI: 10.1103/PhysRevE.83.016603

PACS number(s): 41.20.Jb, 42.70.-a, 42.50.Gy, 42.79.-e

\section{INTRODUCTION}

Transformation optics has led to designs for devices having radical effects on wave propagation, one of the most compelling of which is cloaking [1,2]. The complex material parameters of a transformation optics cloak steer the rays around the region to be hidden, and to a large extent the behavior of the waves mimics that of the rays. However, the literature on such cloaks leaves the impression that transformation optics cloaking produces a decoupling of the waves within and external to the cloaked region (cf. [3-8]). In this paper, we show that on the contrary, rather than being isolated, the cloaked region in fact has a coupling with the environment surrounding the cloak, and this may be amplified by means of carefully chosen parameters within the cloaked region. There is thus a fundamental difference between cloaking for rays and cloaking for waves. We emphasize that the cloaking effectiveness can be made arbitrarily close to the ideal cloak, while keeping the sensing effectiveness fixed. Although we focus on scalar equations in the quasistatic and finite frequency regimes, modeling electrostatic [9] and acoustic cloaks [10], the same phenomenon holds with regard to transformation optics cloaking for transversely polarized electromagnetic (EM) waves (e.g., in the cylindrical geometry [11]). Similar considerations allow sensors to be cloaked from observation by static heat flow or certain cases of matter waves $[12,13]$.

For electromagnetism in the quasistatic regime, it was already shown in [9] that the mean voltage on the exterior surface of an ideal cloak can be measured anywhere inside the cloaked region. At nonzero frequencies, ideal cloaking [2] is accompanied by perfect shielding, meaning that an observer or device within the cloaked region cannot detect any information about the incident wave [11]. Furthermore, approximate cloaking is also accompanied by approximate shielding [5,13] except near certain exceptional frequencies (Neumann eigenvalues of the interior), for which the resulting

*guhlmann@math.uci.edu, gunther@math.washington.edu resonance simultaneously destroys both the cloaking and shielding effects [13].

Here, we consider waves modeled by the Helmholtz equation $\nabla \cdot \sigma(x) \nabla u(x)+\kappa(x) \omega^{2} u(x)=0$ in three dimensions. In electrostatics, $\omega=0$ and $\sigma$ denotes the conductivity; in acoustics, $\omega>0$, and $\sigma$ and $\kappa$ correspond to the inverse of the anisotropic mass density and the inverse of the bulk modulus, respectively. For waves governed by Helmholtz, we describe a sensor effect, in which the combination of an approximate cloak and an inner layer implementing a carefully chosen Robin boundary condition on its inner surface results in effective cloaking but degraded shielding, so that the lowest harmonics of incident waves penetrate deeply into the cloaked region (see Fig. 1). The sensor effect occurs close to but not at interior resonances, and constitutes a new phenomenon. In terms of the design parameters, there are three regimes: generic (standard) cloaking, resonance, and the sensor effect [see Fig. 2 (top)]. The double peak in Fig. 2 (bottom) at $\alpha=17.4$ corresponds to the deterioration of both cloaking and the sensor effect at a resonance; the single dip to its right at $\alpha=18.1$ corresponds to the sensor mode at which cloaking is actually improved.

In [3], Alù and Engheta showed how a plasmonic coating can be used to render an electromagnetic sensor almost invisible to detection by incident waves, while allowing the sensor to remain effective as a device for measuring those very waves. We exploit the enhanced coupling effect described above to show that transformation optics cloaking also allows for cloaked sensors, with a sensor embedded inside the cloaked region efficiently measuring the incident waves without markedly altering them. In contrast to the plasmon-based technique of [3], this method works well for objects quite large compared with the wavelength [cf. Fig. 1 (bottom)]. Furthermore, the quality of the approximate cloaking effect is actually improved at a sensor mode, with the higher order harmonics vanishing to third order in the small parameter $\rho$ we use for asymptotics. Plasmonic cloaking is also related to the polarizability theory (cf. [14]). 

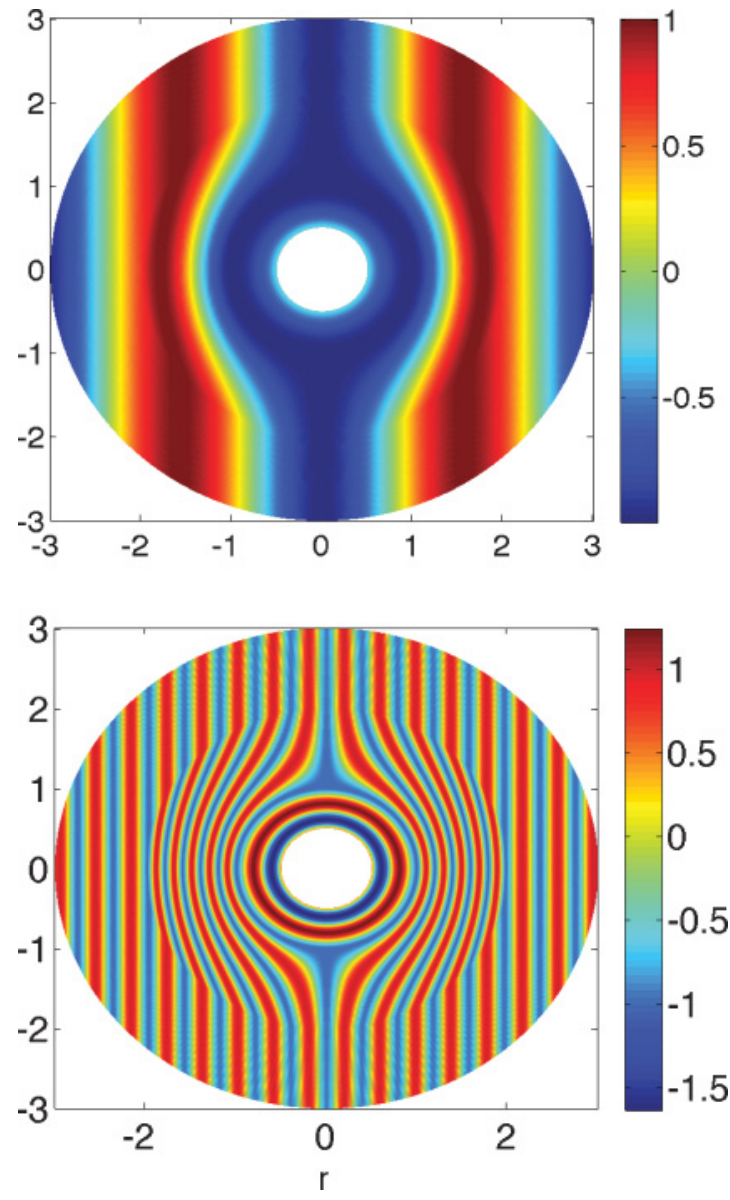

FIG. 1. (Color online) Plane wave incident to the cloaked sensor for frequency $\omega=2$ (top) and $\omega=16$ (bottom). See also animations [15]. In both figures, $\rho=10^{-2}$. Note large magnitude of fields (blue) and their radial nature in spacer layer within the cloaked region.

We remark that our construction, using only right-handed media, is distinct from the anticloak [16], which destroys both cloaking and shielding and requires left-handed materials. The effect described here is also distinct from the sensitivity of the ideal cloak to small perturbations, which degrade cloaking and shielding simultaneously [5], as well as interior resonance effects for approximate cloaks that have been reported previously $[13,17]$. One of the surfaces in our construction is covered with material inducing a real Robin condition, corresponding to an imaginary impedance, $-i \alpha$; cloaking by a thin mantle of such material has been proposed by Alù [18], but this appears to be a different effect than ours. Finally, we note that Sklan [19] also describes penetration of waves into a cloaked region. However, the nature of that phenomenon is quite different from the one described here. Indeed, Ref. [19] (a) shows penetration of momentum into a region infinitesimally close to the cloaking surface (the inner surface of the cloak), corresponding to the gradient of the jump in the pressure field across the surface, while the waves we study occupy the entire cloaked region; (b) deals with ideal cloaking, while the phenomenon we consider occurs only for approximate cloaking and disappears in the ideal limit; and (c) describes effects which occur at any frequency, while the effects described here are parameter sensitive, being due to
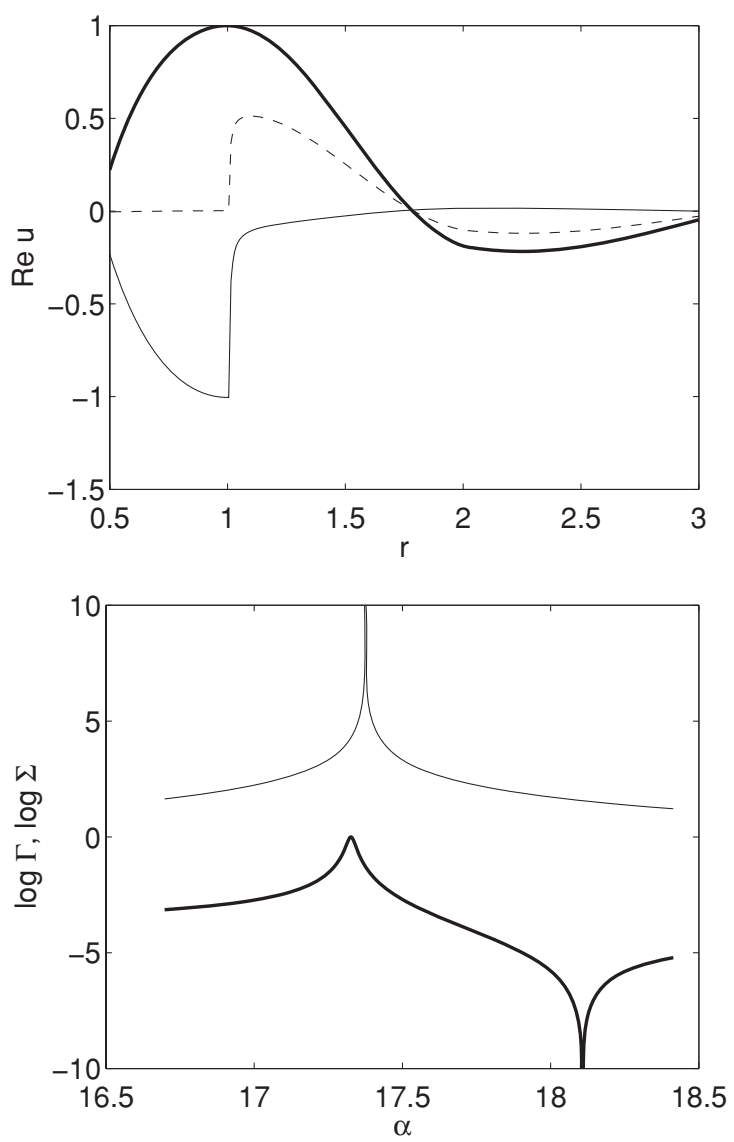

FIG. 2. (Top) Radial solutions $u$ for $\alpha=\alpha^{\text {sen }}$ (thick curve), $\alpha=\alpha^{\text {res }}$ (thin), and for a generic $\alpha$ (dotted). (Bottom) The logarithms of the sensing and scattering, $\log \Sigma$ (thin) and $\log \Gamma$ (thick), as functions of $\alpha$. In both, $\omega=2$ and $\rho=10^{-2}$.

a delicate energy balance near, but not at, interior resonant frequencies.

\section{QUASISTATIC REGIME}

For simplicity, we work throughout with three-dimensional (3D), spherical transformation optics cloaking. In the quasistatic regime, the analysis can be based on ideal cloaking for electrostatics [9]. The solutions $u$ for the ideal cloak considered in [9] are the limits of solutions for more realizable approximate cloaks. Let $B_{R}$ and $S_{R}$, respectively, denote the $3 \mathrm{D}$ ball of radius $R$ centered at the origin $O$, and its boundary, the two-dimensional (2D) sphere. For any $0<R_{1}<R_{2}$, transformation optics [see the transformation $F_{1}$ in (1) below for $R_{1}=1, R_{2}=3$ ] specifies an inhomogeneous, anisotropic cloaking conductivity $\sigma$ on the 3D spherical shell $B_{R_{2}}-B_{R_{1}}$, such that for any passive object (i.e., one without sources or sinks in $B_{R_{1}}$ ), the boundary measurements of electrostatic potentials $u$ at the outer boundary $S_{R_{2}}$ coincide with those made when $B_{R_{2}}$ is filled with a homogeneous, isotropic conductivity $\sigma_{0}$, yielding a perfect cloaking effect. Given a boundary voltage distribution $f$ on $S_{R_{2}}$, the potential $u_{0}$ corresponding to $\sigma_{0}$ takes a value at the origin, $u_{0}(O)=\int_{S_{R_{2}}} f d S$, where $d S$ is normalized surface measure. The potential $u$ corresponding to the same voltage $f$, but with the object in $B_{R_{1}}$ surrounded by 
the cloak $\sigma$, is constantly equal to $u_{0}(O)$ on $B_{R_{1}}$ [9]. Thus, even for the ideal quasistatic cloak, the cloaked region is not electrically isolated, or shielded, from its environment; rather, any passive sensor in $B_{R_{1}}$ measures the mean voltage at $S_{R_{2}}$, while not affecting the potential outside of $B_{R_{1}}$.

\section{FINITE FREQUENCY}

At finite frequency $\omega>0$, the same singular transformation $F_{1}$ from virtual to physical space, applied to homogeneous, isotropic mass density, and isotropic bulk modulus having inverses $\left(\sigma_{0}, \kappa_{0}\right)$, yields an ideal cloak corresponding to $(\sigma, \kappa)$ as in $[2,10]$. Analysis of finite energy waves on the entire region $B_{R_{2}}$ was given in [11], both for the Helmholtz equation and Maxwell's equations; we consider only the former. At frequency $\omega$, the wave $u$ within $B_{R_{1}}$ can be an arbitrary eigenfunction with Neumann (i.e., perfectly reflecting, boundary condition at $S_{R_{1}}$ ). Thus, $u$ must vanish if $\omega$ is not a Neumann eigenfrequency of $B_{R_{1}}$, yielding an idealized decoupling of the cloaked region $B_{R_{1}}$ from $B_{R_{2}}-B_{R_{1}}$. This decoupling is stable, resulting in the cloaking remaining highly effective, even when the ideal cloak $(\sigma, \kappa)$ is replaced by more physically realistic approximate cloaks (see [13]). On the other hand, if $\omega$ is an eigenfrequency, the approximate cloak supports almost trapped states (i.e., resonances), which have very small amplitude near $S_{R_{2}}$ while being almost equal to eigenfunctions of $B_{R_{1}}$ on $B_{R_{1}}$ [13].

We show below that one can create a device containing an almost cloaked yet highly sensitive sensor, consisting of three parts: For $1<R<2$ to be chosen, we form an approximate cloak in the spherical shell $B_{2}-B_{R}$, which tends toward the ideal cloak as $R \searrow 1$. It is convenient to express parameters and asymptotics in terms of the small quantity $\rho:=2(R-1)$, which $\searrow 0$ as $R \searrow 1$. Surround this approximate cloak by a spherical shell $B_{3}-B_{2}$ having $\sigma=\kappa=1$, on whose outer surface $S_{3}$ the external waves will be incident. For a value of $R_{0}<1$, also to be chosen, we then embed a sensing element in the ball $B_{R_{0}}$, clad with a surface whose (complex) impedance induces a real Robin boundary condition, $\partial_{v}+\alpha u=0$ on $S_{R_{0}}$, with $\alpha$ a real parameter to be chosen. Finally, between the innermost two components, we insert a layer $B_{R}-B_{R_{0}}$, which, for simplicity, we take to have $\sigma=\kappa=1$. Another variant of the previous construction occurs when, instead of an obstacle $B_{R_{0}}$ with Robin boundary condition, place inside the cloak (i) a smaller obstacle $B_{R_{0}^{\prime}}$ with a Dirichlet (sound-soft) boundary condition; and (ii) a spacer layer $R_{0}^{\prime}<r<R_{0}$ of homogeneous and isotropic material with $\sigma=1$ and $\kappa$ being a constant $\kappa_{0}$ chosen so that the radially symmetric solution of the obtained equation satisfies $\partial_{r} u\left(R_{0}\right)+\alpha u\left(R_{0}\right)=0$. With this Dirichlet obstacle and spacer layer included, the analysis below is valid mutatis mutandis. In acoustics, such a Dirichlet obstacle corresponds to a sound-soft surface (i.e., a freely moving boundary). If one places inside the ball $B\left(R_{0}^{\prime}\right)$ a device which measures the Neumann boundary value of the solution on the sound-soft boundary $\partial B_{R_{0}^{\prime}}$ [i.e., the normal component of the moment of the surface having the velocity $\vec{V}(\mathbf{x}, t)=\sin (\omega t) \nabla u(\mathbf{x})]$, then this may be measured using another modality, say optically, resulting in an approximately cloaked sensor which can measure $u_{0}(O)$ without significant energy loss.
For the device considered earlier, we first show that $R_{0}$ and $\alpha=\alpha^{\text {res }}(\rho)$ may be chosen to allow the total device to support a resonant wave $\widetilde{u}$, of very small amplitude in $B_{3}-B_{R}$ and almost equal to a central (i.e., rotationally symmetric) Robin-Neumann eigenfunction $\tilde{u}_{0}$ of unit energy in the spacer $B_{R}-B_{R_{0}}$. Moreover, we show that by varying $\alpha^{\text {res }}(\rho)$ to a nearby value $\alpha^{\operatorname{sen}}(\rho)$ with $\alpha^{\text {res }}(\rho)-\alpha^{\operatorname{sen}}(\rho) \simeq b_{o} \rho$ as $\rho \searrow 0$, the resonance effect is changed into a sensor effect: the resulting wave is proportional, at $r=R_{0}$, to the value $u_{0}(O)$ that the wave would take at the origin in the absence of the device.

The sensor is nevertheless effectively cloaked from an outside observer, since the Dirichlet-to-Neumann map on $S_{3}$ (i.e., the operator mapping the Dirichlet boundary value $u$ on $S_{3}$ to the Neumann boundary value $\partial_{v} u$ on $S_{3}$ ), remains equal to the free space Dirichlet-to-Neumann map, up to a perturbation order $O\left(\rho^{2}\right)$.

\section{ANALYSIS OF APPROXIMATE CLOAKS}

To see this in more detail, we recall some facts concerning nonsingular approximations to ideal cloaks. To start, let $1 \leqslant R<2$, and set $\rho=2(R-1), 0 \leqslant \rho<2$, so that $R \searrow 1$ as $\rho \searrow 0$. Introduce the coordinate transformation $F_{R}: B_{3}-$ $B_{\rho} \rightarrow B_{3}-B_{R}$,

$$
\mathbf{x}:=F_{R}(\mathbf{y})=\left\{\begin{array}{cll}
\mathbf{y}, & \text { for } & 2<|\mathbf{y}|<3 \\
\left(1+\frac{|\mathbf{y}|}{2}\right) \frac{\mathbf{y}}{|\mathbf{y}|}, & \text { for } & \rho<|\mathbf{y}| \leqslant 2 .
\end{array}\right.
$$

For $R=1(\rho=0)$, this is the singular transformation of [2,9], leading to the ideal transformation optics cloak, while for $R>1(\rho>0), F_{R}$ is nonsingular and leads to a class of approximate cloaks $[5,13,17]$. Thus, if $\sigma_{0} \equiv \delta^{j k}$ denotes the homogeneous, isotropic tensor, then for $R>1$ the transformed tensor $\sigma^{j k}(\mathbf{x})$ is nonsingular on $R<|\mathbf{x}| \leqslant 2$ [i.e., its eigenvalues are bounded from above and below (with, however, the lower bounds of two of them going to 0 as $R \searrow 1$ )]. We then define the approximate cloak tensor $\sigma_{R}$ on $B_{3}$ as

$$
\sigma_{R}^{j k}(\mathbf{x})=\left\{\begin{array}{cll}
\delta^{j k}, & \text { for } & 2<|\mathbf{x}|<3 \\
\sigma^{j k}(\mathbf{x}), & \text { for } & R<|\mathbf{y}| \leqslant 2, \\
\delta^{j k}, & \text { for } & |\mathbf{x}|<R
\end{array}\right.
$$

Here, $\sigma(x)=(D F(x)) \sigma_{0}(x)(D F(x))^{t} / \operatorname{det}(D F(x))$ is the standard cloaking tensor. Define also

$$
\kappa_{R}(\mathbf{x})=\left\{\begin{array}{ccc}
1, & \text { for } & 2<|\mathbf{x}|<3, \\
64|\mathbf{x}|^{-4}(|\mathbf{x}|-1)^{4}, & \text { for } & R<|\mathbf{x}| \leqslant 2, \\
1, & \text { for } & |\mathbf{x}|<R
\end{array}\right.
$$

Now place inside the cloak a scatterer $B_{R_{0}}$ of radius $R_{0}<1$, with a surface whose impedance induces a real Robin boundary condition on the sphere $S_{R_{0}}$. Thus, we consider in the domain $\Omega=B_{3}-B_{R_{0}}$ the solutions of the problem,

$$
\begin{gathered}
\left(\nabla \cdot \sigma_{R} \nabla+\omega^{2} \kappa_{R}\right) u_{R}=0, \quad \text { in } \Omega, \\
\left.u_{R}\right|_{S_{3}}=f,\left.\left(\partial_{r}+\alpha\right) u_{R}\right|_{S_{R_{0}}}=0,
\end{gathered}
$$

for an impedance $\lambda=-i \alpha$ to be specified later. As $\sigma_{R}$ and $\kappa_{R}$ are now nonsingular everywhere, across the internal interface $S_{R}$ we have the standard transmission conditions,

$$
\begin{aligned}
\left.u_{R}\right|_{R_{R^{+}}} & =\left.u_{R}\right|_{S_{R^{-}}}, \\
\left.\mathbf{e}_{\mathbf{r}} \cdot \sigma_{R} \nabla u_{R}\right|_{S_{R^{+}}} & =\left.\mathbf{e}_{\mathbf{r}} \cdot \sigma_{R} \nabla u_{R}\right|_{S_{R^{-}}},
\end{aligned}
$$


where $\mathbf{e}_{\mathbf{r}}$ is the radial unit vector and \pm indicates the trace on $S_{R}$ as $r \rightarrow R^{ \pm}$. We note that the approximate cloak $\left(\sigma_{R}, \kappa_{R}\right)$ with anisotropic density tensor $\sigma_{R}^{-1}$ can also be approximated, with arbitrary precision, by a cloak with isotropic density [13].

In the physical space $\Omega$ we have

$$
u_{R}(\mathbf{x})=\left\{\begin{array}{cll}
v_{R}^{+}\left(F_{R}^{-1}(\mathbf{x})\right), & \text { for } & R<|\mathbf{x}|<3, \\
v_{R}^{-}(\mathbf{x}), & \text { for } & R_{0}<|\mathbf{x}| \leqslant R,
\end{array}\right.
$$

with $v_{R}^{ \pm}$in the virtual space, which consists of the disjoint union $\left(B_{3}-B_{\rho}\right) \cup\left(B_{R}-B_{R_{0}}\right)$, satisfying

$$
\begin{array}{r}
\left(\nabla^{2}+\omega^{2}\right) v_{R}^{+}(\mathbf{y})=0 \text { for } \rho<|\mathbf{y}|<3,\left.v_{R}^{+}\right|_{S_{3}}=f, \\
\left(\nabla^{2}+\omega^{2}\right) v_{R}^{-}(\mathbf{y})=0, R_{0}<|\mathbf{y}|<R,\left.\left(\partial_{r}+\alpha\right) v_{R}^{-}\right|_{S_{R_{0}}}=0 .
\end{array}
$$

With respect to spherical coordinates $(r, \theta, \varphi)$, the transmission conditions (5) become $v_{R}^{+}(\rho, \theta, \phi)=v_{R}^{-}(R, \theta, \phi)$ and $\rho^{2} \partial_{r} v_{R}^{+}(\rho, \theta, \phi)=R^{2} \partial_{r} v_{R}^{-}(R, \theta, \phi)$. Since $\sigma_{R}, \kappa_{R}$ are spherically symmetric, we can separate variables in (4), representing $u_{R}$ as

$$
u_{R}(r, \theta, \phi)=\sum_{n=0}^{\infty} \sum_{m=-n}^{n} u_{R}^{n, m}(r) Y_{n}^{m}(\theta, \phi),
$$

where $Y_{n}^{m}$ are the standard spherical harmonics. Then Eq. (4) gives rise to a family of boundary value problems for the $u_{R}^{n, m}$. For our purposes, the most important one is for the lowest harmonic term, $u_{R}^{0,0}$ (i.e., the radial component of $u_{R}$ ), which is independent of $(\theta, \phi)$. This is studied in the next section.

\section{LOWEST HARMONIC AND THE SENSOR EFFECT}

Consider the problem (4) with $f=$ const $=j_{0}(3 \omega)$, so that $u_{R}^{\alpha}(r, \theta, \phi)=u_{R}^{\alpha}(r)$, and express asymptotics of the waves in terms of $\rho \searrow 0$. Analysis of the solutions reveals three distinct regimes, depending on the Robin coefficient $\alpha$. This can be seen informally, building up the wave inward, from the exterior of the cloak, through the cloak, and into the cloaked sensor region, as follows. On the spherical shell $\{2 \leqslant r \leqslant 3\}$, a radial solution of (4) solves the ordinary differential equation,

$$
\left(r^{2} u^{\prime}(r)\right)^{\prime}+\omega^{2} r^{2} u(r)=0 .
$$

An incident wave has Cauchy data (i.e., the value of the wave and its normal derivative) $\left(u(3), u^{\prime}(3)\right)$. Using this as initial conditions for (9) at the right endpoint of $\{2 \leqslant r \leqslant 3\}$, one then finds the Cauchy data at the left endpoint, $\left(u\left(2^{+}\right), u^{\prime}\left(2^{+}\right)\right)$. Applying the transmission conditions (5), one then finds $\left(u\left(2^{-}\right), u^{\prime}\left(2^{-}\right)\right)$. Continuing inward through the approximate cloak, we solve the initial value problem with this Cauchy data for

$$
\left((r-1)^{2} u^{\prime}(r)\right)^{\prime}+4 \omega^{2} r^{2} u(r)=0, \quad R \leqslant r \leqslant 2,
$$

cf. $[9,11]$, evaluate the Cauchy data at $r=R^{+}$, and then use (5) again to find $\left(u\left(R^{-}\right), u^{\prime}\left(R^{-}\right)\right)$. In the spacer layer $\left\{R_{0} \leqslant r \leqslant R\right\}, u$ satisfies (9), with initial conditions at $r=R^{-}$ determined by the previous procedure, so that finally we may evaluate $\left(u\left(R_{0}\right), u^{\prime}\left(R_{0}\right)\right)$. Starting with $\left(u(3), u^{\prime}(3)\right)=(0,1)$, $\alpha=\alpha^{\text {res }}(\rho):=-u^{\prime}\left(R_{0}\right) / u\left(R_{0}\right)$ gives a value of the impedance which induces a resonant, or trapped, wave, $u^{\text {res }}$. On the other hand, starting with $\left(u(3), u^{\prime}(3)\right)=\left(j_{0}(3 \omega), \omega j_{0}^{\prime}(3 \omega)\right)$ gives $\alpha=$ $\alpha^{\text {sen }}(\rho):=-u^{\prime}\left(R_{0}\right) / u\left(R_{0}\right)$ corresponding to the sensor effect and giving rise to the sensor solution, $u^{\text {sen }}$. A calculation shows that $\alpha^{\text {sen }}$ is close to $\alpha^{\text {res}}$ : for some $b_{0} \neq 0$, one has $\alpha^{\text {res }}(\rho)-\alpha^{\operatorname{sen}}(\rho)=b_{0} \rho+O\left(\rho^{2}\right)$, as $\rho \searrow 0$. Thus, the sensor effect is quite sensitive to perturbations.

More careful analysis confirms the following three regimes, depending on how close $\alpha$ is to $\alpha^{\text {sen }}(\rho)$ :

(i) Cloaking for generic impedance. If $\alpha$ is bounded away from $\alpha^{\text {sen }}$ (i.e., $\left|\alpha-\alpha^{\operatorname{sen}}(\rho)\right| \geqslant c>0$ ), or even when $\alpha=\alpha^{\text {sen }}(\rho)+b \rho^{s}$ for some $0<s<1, b \neq 0$, then the cloak acts as an effective approximate cloak, and the field goes to zero in the cloaked region as $\rho \searrow 0$, so that there is no sensor effect.

(ii) Resonance effect. For the specific value $\alpha=\alpha^{\text {res }}(\rho)$, the interior resonance leads to both the destruction of cloaking and the absence of shielding, since then $\omega$ is an eigenfrequency of Eq. (4) with boundary condition $u=0$ on $\partial \Omega$ and $\left(\partial_{r}+\alpha\right)$ $u=0$ on $r=R_{0}$.

(iii) Cloaking with sensor effect. For the value $\alpha=\alpha^{\text {sen }}(\rho)$, the cloak acts as an effective approximate cloak, but inside the cloaked region the solution is proportional to the value which the field would have had at the origin in free space, with proportionality of order $O(1)$ as $\rho \rightarrow 0$, that is, for $R_{0}<r<$ $R, u_{R}(r)=c^{0}(r) v_{0}+O(\rho)$, where $c^{0}(r)$ is a not identically vanishing function and $v_{0}$ is the value at $O$ of the solution to the free-space $\nabla \cdot \nabla v+\omega^{2} v=0,\left.v\right|_{r=3}=f$. Thus, in the sensor mode the cloak functions as an "invisible magnifying glass," where the value which the field would have had in the empty space at a single point can be measured anywhere inside the cloak. This allows one to enclose a measurement device which does not affect the incident fields being measured.

If $u^{\text {res }}(r, \rho)$ is the resonance solution and $u^{\text {cloak }}(r, \rho)$ is a general cloaking solution [i.e., they are the radial solutions of (7) corresponding to $\alpha=\alpha^{\text {res }}(\rho)$ and a generic Robin coefficient $\alpha=\alpha^{\text {cloak }}$ bounded away from $\alpha^{\text {sen }}(\rho)$ ], the radial sensor solution $u^{\text {sen }}(r, \rho)$ can be obtained as a linear combination $\beta_{1} u^{\text {res }}(r, \rho)+\beta_{2} u^{\text {cloak }}(r, \rho)$ where $\beta_{1}, \beta_{2} \in \mathbb{R}$ are chosen so that the scattering vanishes, and $\alpha^{\operatorname{sen}}(\rho)$ is obtained by computing the suitable Robin coefficient for this particular solution. Roughly speaking, when $\alpha=\alpha^{\text {sen }}(\rho)$, the frequency $\omega$ is so close to the eigenfrequency of the inside of the cloak that the energy flux from the inside to outside and from the outside to inside through the surface $r=R$ are balanced. The solution inside the cloak does not blow up and the energy flux from the inside cancels the scattering caused by the fact that the cloak is only an approximate cloak, not a perfect cloak. Animations of the three regimes, are provided in [15] with $\rho=10^{-2}, \omega=16$. For the sensor effect, $\alpha=-56.0$, and for the generic cloaking, $\alpha=2$, we solve the scattering problem in $\mathbb{R}^{3}$ with an incident plane wave and use $0 \leqslant n \leqslant 70$ in (8). For the resonance effect, $\alpha=-56.1$ and $\left.u_{R}\right|_{S_{3}}=0$.

We now analyze these effects quantitatively. As a wave incident to the cloak can be written in terms of Bessel functions $j_{n}(\omega r)$ and the scattered wave in terms of Hankel functions $h_{n}^{(1)}(\omega r)$, the scattering effect for the zeroth order harmonic can be numerically measured by the ratio $\Gamma=\left|\frac{c_{0}^{s c}}{c_{0}^{i n}}\right|$, where the zeroth order harmonic of the total field is $c_{0}^{i n} j_{0}(\omega r)+c_{0}^{s c} h_{0}^{(1)}(\omega r)$. Cloaking then corresponds to $\Gamma \ll 1$, while the breakdown of cloaking takes place when $\Gamma=O(1)$. Similarly, quantify the shielding effect by 
defining $\Sigma=\left|u_{R}^{\alpha}\left(R_{0}\right)\right| /\left|u_{R}^{\alpha}(3)\right|$, so that $\Sigma \ll 1$ corresponds to effective shielding of the cloaked region from the incident waves; $\Sigma \gg 1$ corresponds to the resonance regime; and the sensor effect corresponds to the case when $\Sigma$ stays between positive constants as $\rho \rightarrow 0$ so that the field inside the cloaked region gives adequate information about the external field.

A parameter $\alpha$ with $\left|\alpha-\alpha^{\text {res }}(\rho)\right|>c_{0}>0$, or even with $\left|\alpha-\alpha^{\text {res }}(\rho)\right|>c_{1} \rho^{s}, c_{1}>0$, for some $0<s<1$, corresponds to the standard effects of both cloaking and shielding. The values of $\alpha$ near $\alpha=\alpha^{\text {res }}$, namely $\alpha-\alpha^{\text {res }}=O\left(\rho^{2}\right)$, correspond to the resonance effect, with $\Sigma=O\left(\rho^{-1}\right)$. However, a new phenomenon appears in the region near $\alpha=\alpha^{\text {sen }}$, namely $\alpha(\rho)=\alpha^{\text {res }}+b_{0} \rho+O\left(\rho^{2}\right)$. In this region $\Gamma=O\left(\rho^{2}\right)$ while $\Sigma=O(1)$ (i.e., cloaking is effective but shielding is degraded). Moreover, for the higher order spherical harmonic components corresponding to $Y_{n}^{m}(\theta, \phi)$ we can introduce figures of merit $\Gamma_{n m}(\rho)$ and $\Sigma_{n m}(\rho)$ similar to $\Gamma, \Sigma$, respectively. Then, for $n \geqslant 1$,

$$
\Gamma_{n m}(\rho)=O\left(\rho^{(2 n+1)}\right), \quad \Sigma_{n m}(\rho)=O\left(\rho^{(n+1)}\right),
$$

showing that, for the higher harmonics there is both a strong shielding effect (i.e., lack of sensor effect) and an almost ideal cloaking. Comparing the formulas previously mentioned with the situation for $n=0$, we see that the effect of the higher harmonics is comparatively negligible: one still has almost ideal cloaking and sensor effects. Hence, if a measurement device inside the obstacle $B_{R_{0}}$ implements this Robin boundary condition, then the device can measure the mean value of the wave on the boundary $S_{R_{0}}$ of the obstacle. Thus, an observer inside the cloaked region is able to measure the outside fields, while these measurements do not disturb the field outside the cloaking device. Observe that the cloaking and the sensing at $\alpha=\alpha^{\operatorname{sen}}(\rho)$ is effectively independent of $\rho$, as $\rho \searrow 0$. Recall that the cloak in the sensor mode operates with time-harmonic waves with given frequency $\omega$. Thus, if a device producing a time-harmonic source is activated outside the cloak, there is a delay before the wave reaches its time-harmonic steady state and the zeroth order component fully penetrates the cloak. Since the approximate cloak corresponds to a small object in the virtual space [17], which has a small cross section, the smaller $\rho$ is the less energy is transferred into the cloaked region, and the longer it takes before the wave reaches its sensor mode state there. This explains why the sensor effect disappears in the case of ideal cloaking; indeed, in this case the time delay becomes infinite. Finally, we again note that the fact that $\alpha^{\text {res }}(\rho)-\alpha^{\text {sen }}(\rho)=O(\rho)$, shows that the sensor and resonance effect is highly sensitive to the value of $\alpha$. By readjusting $\alpha$, an interior observer can tune this sensor to any desired frequency $\omega$.

\section{ANALYSIS OF ENERGY LOSS}

Finally, since measuring the wave on the Robin-boundary $\partial B_{R_{0}}$ (or on the Dirichlet-boundary $\partial B_{R_{0}^{\prime}}$ if we use the spacer layer and a sound soft obstacle) will result in some energy loss, we analyze how this loss affects the sensing and shielding. To this end, we compute the solutions in the case when the real Robin coefficient $\alpha$ has been replaced by a complex one, $\alpha+i \beta$. In the numerical simulations $\alpha$ is tuned
TABLE I. Sensing and cloaking figures of merit in the presence of energy loss.

\begin{tabular}{lcc}
\hline \hline$\beta$ & $\Sigma$ & $\Gamma$ \\
\hline 0 & 4.77 & $4.71 \times 10^{-16}$ \\
$\beta_{1}$ & 3.83 & 0.0111 \\
$\beta_{2}$ & 2.67 & 0.0158 \\
\hline \hline
\end{tabular}

so that with the frequency $\omega=2$ and $\rho=10^{-2}$ we have sensor effect, that is, $\alpha=\alpha^{\operatorname{sen}}(\rho)=18.1$. We considered two values of $\beta$, namely when $\beta_{1}=3 \times 10^{-2} \alpha^{\text {sen }}(\rho)$ and $\beta_{2}=6 \times 10^{-2} \alpha^{\text {sen }}(\rho)$, that is, the imaginary part is either $3 \%$ or $6 \%$ of the real part. Values of $\Sigma$ (sensing) and $\Gamma$ (scattering) in Table I correspond to the solutions in Fig. 3. As seen in Fig. 3 and the table, increasing energy loss starts to degrade the sensing effect, but the scattering does not grow very much. Thus, even for the parameter value $\rho=10^{-2}$ for which the cloaking effect is relatively strong, the energy loss does not destroy the cloaking property. However, due to energy loss the magnitude of the field becomes smaller inside the cloak, and thus on the interior boundary. Thus, in the presence of energy loss, the field needs to be measured with a more sensitive device.

\section{DISCUSSION}

We have described a new effect for transformation-opticsbased cloaking. In the quasistatic regime, it was previously known that the mean voltage on the exterior surface of the cloak can be measured anywhere inside the cloaked region [9]. On the other hand, at nonzero frequencies, ideal cloaking [1,2] is accompanied by shielding [11]: There is a decoupling of the fields inside and outside of the cloaked region, so that external observations do not provide any indication of the presence of a cloaked object, nor is any information about the fields outside detectable inside the cloaked region. Approximate shielding goes hand in hand with approximate cloaking, with the shielding improving as the cloaking does, except at resonant frequencies, at which both are destroyed [13]. We have described a sensor effect that breaks this connection

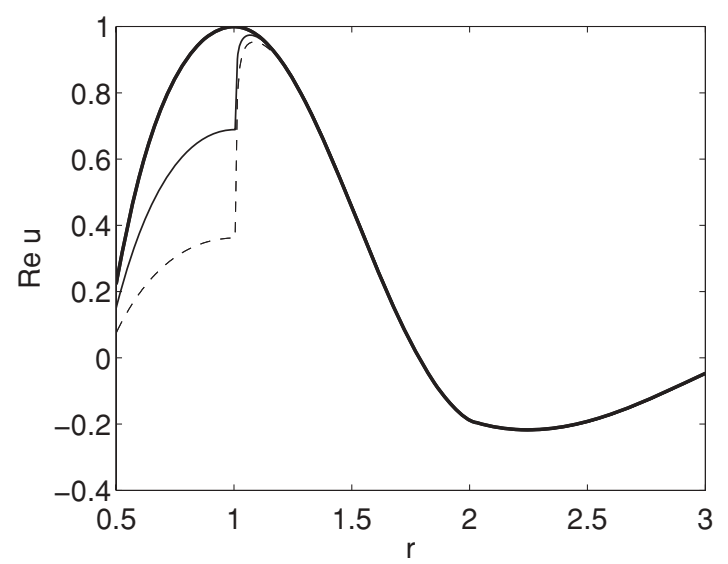

FIG. 3. Total fields corresponding to a plane wave scattering from the cloaked sensor for frequency $\omega=2, \rho=10^{-2}$, and Robin coefficient $\alpha^{\text {sen }}$ (thick curve), $\alpha^{\text {sen }}+i \beta_{1}$ (solid thin), and $\alpha^{\text {sen }}+i \beta_{2}$ (dashed thin). 
between cloaking and shielding, allowing the former without the latter, showing that transformation optics permits sensors to be cloaked. This effect occurs close to, but not at resonance, and is a distinct phenomenon. The sensor effect exists for any type of wave governed by (or reducible to) the Helmholtz equation, including scalar electromagnetism, acoustics and matter waves. It would be interesting to understand to what extent it holds for other systems, such as Maxwell's equations. Our construction likely generalizes to Maxwell for 3D cloaks in the cylindrical geometry. However, as Maxwell's equations do not have spherically symmetric solutions, the extension of the sensor effect to the spherical geometry remains unclear.

\section{ACKNOWLEDGMENTS}

A.G. and G.U. are supported by U.S. National Science Foundation, Y.K. by UK Engineering and Physical Sciences Research Council, M.L. by Academy of Finland (Project No. CoE-213476), and G.U. by a Walker Family Endowed Professorship at University of Washington.
[1] U. Leonhardt, Science 312, 1777 (2006); U. Leonhardt and T. Tyc, ibid. 323, 110 (2009).

[2] J. B. Pendry, D. Schurig, and D. R. Smith, Science 312, 1780 (2006).

[3] A. Alù and N. Engheta, Phys. Rev. Lett. 102, 233901 (2009).

[4] S. Cummer, B. Popa, D. Schurig, and D. Smith, Phys. Rev. E 74, 036621 (2006).

[5] Z. Ruan, M. Yan, C. Neff, and M. Qiu, Phys. Rev. Lett. 99, 113903 (2007).

[6] J. McGuirk and P. Collins, Opt. Express 16, 17560 (2008).

[7] G. Castaldi et al., Opt. Express 17, 3101 (2009).

[8] F. García de Abajo, Physics 2, 47 (2009).

[9] A. Greenleaf, M. Lassas, and G. Uhlmann, Physiolog. Meas. 24, 413 (2003); Math. Res. Lett. 10, 685 (2003).

[10] H. Chen and C. T. Chan, Appl. Phys. Lett. 91, 183518 (2007); S. Cummer et al., Phys. Rev. Lett. 100, 024301 (2008);

A. Greenleaf et al., e-print arXiv:0801.3279v1.
[11] A. Greenleaf, Y. Kurylev, M. Lassas, and G. Uhlmann, Commun. Math. Phys. 275, 749 (2007).

[12] S. Zhang, D. Genov, C. Sun, and X. Zhang, Phys. Rev. Lett. 100, 123002 (2008).

[13] A. Greenleaf, Y. Kurylev, M. Lassas, and G. Uhlmann, Phys. Rev. Lett. 101, 220404 (2008); New J. Phys. 10, 115024 (2008); e-print arXiv:0812.1706.

[14] I. Lindell, A. Sihvola, S. Tretyakov, and A. Viitanen, Electromagnetics Waves in Chiral and Bi-isotropic Media (Artech House, London, 1994).

[15] See supplemental material at [http://link.aps.org/supplemental/ 10.1103/PhysRevE.83.016603] for three animated simulations of cloaking device in generic, sensor, and resonance regimes.

[16] H. Chen, X. Luo, H. Ma, and C. T. Chan, Opt. Express 16, 14603 (2008).

[17] R. Kohn, D. Onofrei, M. Vogelius, and M. Weinstein, Commun. Pure Appl. Math. 63, 973 (2010).

[18] A. Aù, Phys. Rev. B 80, 245115 (2009).

[19] S. Sklan, Phys. Rev. E 81, 016606 (2010). 